\title{
International data and information exchange in Europe - systems to assist the EU Member States in radiological and nuclear emergency situations
}

\author{
M. De Cort ${ }^{1}$, G. de Vries ${ }^{1}$, S. Galmarini ${ }^{2}$ and V. Tanner ${ }^{3}$ \\ ${ }^{1}$ European Commission, JRC, Institute for Transuranium Elements, 21027 Ispra (VA), Italy \\ 2 European Commission, JRC, Institute for Environment and Sustainability, \\ 21027 Ispra (VA), Italy \\ ${ }^{3}$ European Commission, DG ENER, Radiation Protection Unit, \\ 2920 Luxembourg, Luxembourg
}

\begin{abstract}
During the early phase of a large-scale accident with release of radioactivity to the atmosphere, it is essential to notify and inform competent authorities as early and as extensively as possible. Only when the accident is rapidly notified and information is continuously made available in the form of real-time monitoring data and dispersion forecasts are decision makers able to define appropriate countermeasures. The Chernobyl accident taught us that information exchange should be carried out in a harmonised and consistent manner. Although several European countries already had developed automatic monitoring networks by 1986 and in some cases established bilateral agreements to exchange this information, the size of the accident demonstrated the need to extend such schemes to the continental scale. It became important to have commonly agreed international data formats and procedures in place. Over the past 25 years, the European Commission has invested in improving the rapid exchange of information and data in the event of a major accident. For the early phase of emergency support, it has focussed on three closely related systems: the early notification system ECURIE, the automatic data exchange platform EURDEP and the atmospheric dispersion model exchange and evaluation system ENSEMBLE. Starting from the legal background, we describe these information systems in detail with an emphasis on their current status and their planned future developments.
\end{abstract}

\section{INTRODUCTION}

Supporting the Radiation Protection Unit of the Directorate General for Energy (DG ENER D.4) in Luxembourg, the Radioactivity Environmental Monitoring (REM) group of the Joint Research Centre (JRC) in Ispra, Italy, has more than 15 years of experience in developing systems for making early notification and reliable radiological information available to the EU Member States in the event of a nuclear accident. The legal basis for this is Council Decision 87/600/Euratom of 14 December 1987 on Community arrangements for the early exchange of information in the event of a radiological emergency [1].

The large transboundary effects of the radioactive release during the Chernobyl accident clearly emphasized the need for international provisions to exchange data and information. In particular during the early phase of an accident, information has to be exchanged as soon as it becomes available and preferably in real-time to allow Competent Authorities to take timely and appropriate countermeasures. This is even more the case if during the accident a considerable amount of radioactivity is released into the atmosphere: in short time the radioactive plume can travel over large distances and in any direction, depending on the meteorology during the accident. As a consequence decision makers often have little time available to define appropriate countermeasures before the plume arrives. If, however, a remote accident is rapidly notified and continuous information is available in the form of real-time monitoring data and/or dispersion forecasts, then the decision makers are better equipped to define the most appropriate countermeasures. 
The Chernobyl accident resulted in increased radioactivity levels over most of Europe, and after the accident many additional countries set up GDR-based automatic monitoring networks for delineating the radioactive cloud. The Fukushima accident was however far away so that the maximum air concentration values over Europe were between a factor 1000 to 10000 lower than during the Chernobyl accident. For detecting such low increases of radioactivity the GDR networks are not sensitive enough. Therefore the EC asked the EURDEP data-providers to increase the amount of air-concentration data, i.e. measurements from High Volume Air Samplers (HVAS).

\section{ECURIE}

The European Community Urgent Radiological Information Exchange (ECURIE) system is the technical implementation of the above-mentioned Council Decision 87/600/Euratom [1]. It is the official EU early notification system that alerts the 27 EU Member States, Switzerland, Croatia and the Republic of Macedonia. This requires that in case of a radiological/nuclear emergency when a country decides to take counter-measures in order to protect its population, it must promptly notify the European Commission (EC). After authentication, the EC promptly forwards this notification to all ECURIE Contact Points. Subsequently, the system provides a continuous flow of information during the accident.

The participants to the ECURIE network are the nominated Contact Points (CPs) and Competent Authorities (CAs) of the Member States and the EC. The nominated CPs must be available on a 24hour basis, 7 days a week. At the Commission, the central Security Office in Brussels acts as CP and the Radiation Protection Unit of the Directorate General for Energy (DG ENER D.4) as CA, while the DG JRC in Ispra, Italy, is responsible for developing the ECURIE software (CoDecS) and for giving technical and scientific support. Much effort has been invested in order to assure that the ECURIE network operates reliably: for redundancy there are always two CoDecS stations in operation both at the EC-CP and the EC-CA. In addition the EC-CA uses an appropriate functionality of the CoDecS software that allows a fully automated, daily verification of the CoDecS stations in all the Member States. In addition the DG ENER verifies the availability of the national CPs and CAs through unannounced communication exercises several times per year. Last but not least, the Commission organizes one full accident simulation exercise each year for training the national CAs.

The CoDecS software, developed by REM, assists the ECURIE CAs in creating a notification message and allows them to send and receive these messages. It is entirely written in the Java programming language, uses the Simple Mail Transfer Protocol (SMTP) to transmit messages and has a Graphical User Interface with the look and feel of an Email client. In contrast to standard Email (SMTP) applications that use a store and forward mechanism, the built-in SMTP routine of CoDecS creates a direct (point-to-point) connection between two stations in order to obtain an immediate confirmation of receipt. Each message has a digital signature for authentication and integrity verification purposes and can include attachments.

The CoDecS software will be replaced by a web-based system, called WebECURIE, in 2012. It uses an innovative status-board concept that should facilitate the consultation of the information contained in the system (Figure 1). This status board concept offers four levels of detail. It allows the user to view: 1) detailed information made available by one or more countries; 2) a one-page summary information for each country; 3) a single set of detailed fields that contain information originating from all countries that made information available; and 4) a one-page summary of the most important accident information elements that originate from all the countries. All information shown will be continuously and automatically refreshed, and for every single field the user can optionally see how (and by which authority) the values in a field were updated. The top-level summary page should allow decision makers to stay continuously and easily informed about the most important parameters of the accident but with the possibility to view more detailed information if necessary, while at the same time the experts are able to access all the detailed information. It is expected that this way of presenting information will greatly help decision makers with assimilating large quantities of information from different sources. In 

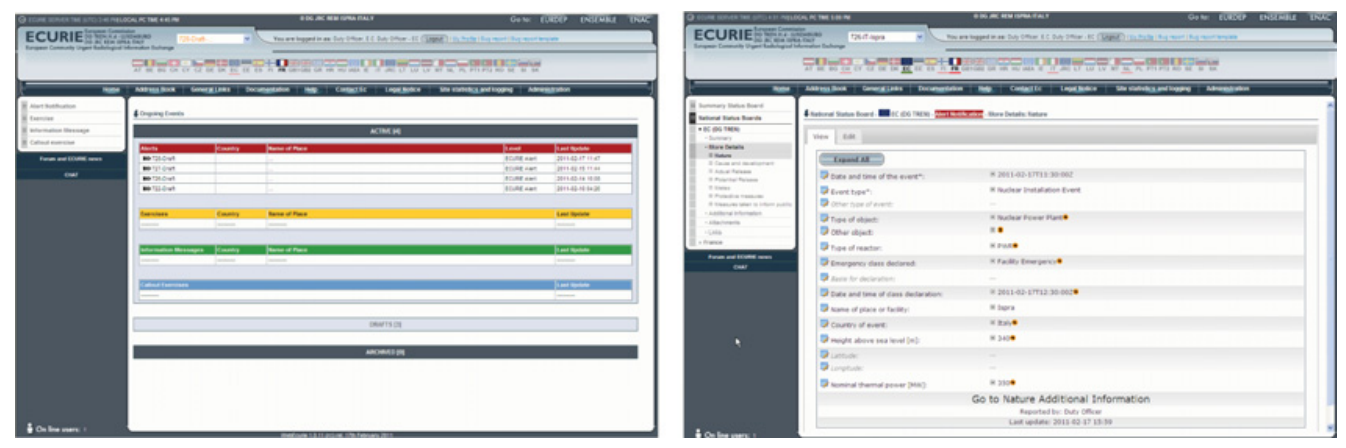

Figure 1. WebECURIE interface: general overview (left) and detailed message content (right).

addition, since this is being developed with web technology, national radiological duty officers will be able to respond to ECURIE messages wherever they have an Internet connection.

A very important recent achievement is the agreement between the IAEA and EC to use a common data format and data-exchange protocol, the International Radiation Information Exchange (IRIX) format. Even better, the IAEA and the EC also developed a common set of data elements that are considered to be relevant during an accident. Both the future IAEA web-site USIE and the future EC web-site WebECURIE will be compatible with the IRIX standards. The major benefits are that messages can be exchanged between ECURIE and ENATOM without loss of information and without the need for conversion and that competent authorities can send information to the EC and to the IAEA using the same protocols and data-format.

The information required from the competent authorities under ECURIE also includes environmental monitoring data. Because both the current and the future ECURIE software require manual input which strongly limits the amount of such data that can be entered, it is the strategy of the EC to exchange the environmental monitoring data by means of a dedicated and automated system, called EURDEP.

\section{EURDEP}

The EURDEP system has been designed to facilitate transmission of large datasets from environmental monitoring networks, thereby taking away the burden from national crisis centres to manually report and transmit such data. The initial objectives of this project in 1994 were to obtain "voluntary participation of European countries in rapid exchange of radiological information throughout Europe in order to support nuclear emergency response, and the exploration, without prior commitment, of its feasibility for meeting relevant EC requirements in routine and emergency conditions" (resp. Article 36 of the Euratom Treaty, Council Decision 87/600 Euratom).

The outcomes of the initial objectives were a data-exchange format and a data-exchange network. The EURDEP data-format has become a de-facto standard in Europe for the exchange of radiological monitoring data on an international level. In addition the data-format is used by various countries for data-exchange on national and bi-lateral levels (e.g. by the Baltic Sea States).

Most of the participating organizations make their data available continuously on an hourly basis. The collected data can be analysed and accessed by means of a simple Geographical Information System (GIS) interface, which is available via the EURDEP web site (Figure 2), http://eurdep.jrc.ec.europa.eu. Most of the data on this site are also accessible to the general public.

Currently the EURDEP network continuously (during routine and emergency) makes data available from 37 organizations in 35 European countries. Most data originate from some 4400 automatic gamma dose-rate stations and are refreshed every hour. In addition air concentration data from some 100 

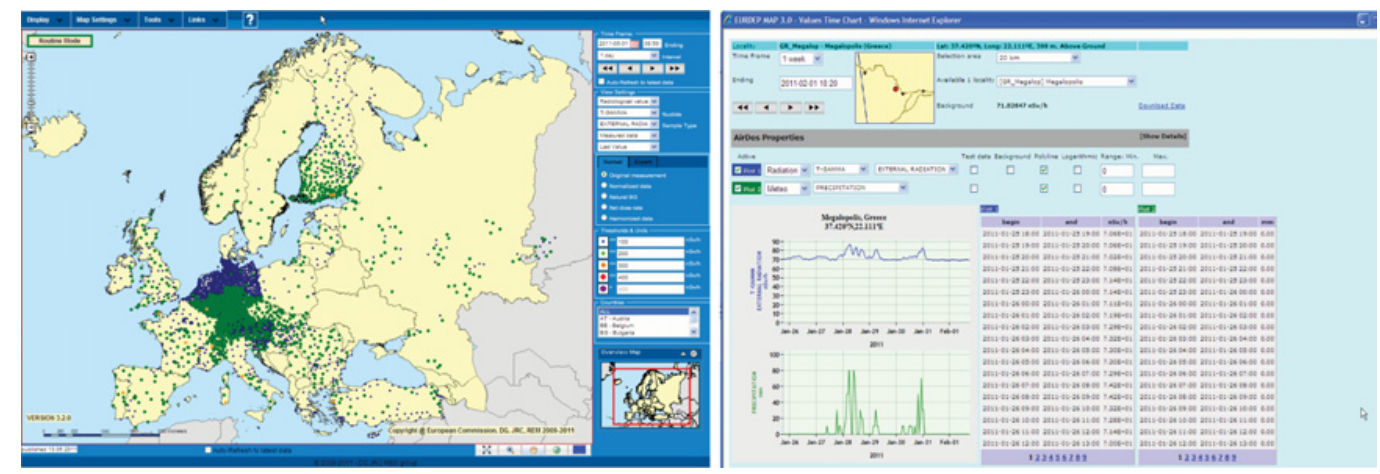

Figure 2. EURDEP web interface: the general overview map (left) and detailed radiation and meteo information of a selected monitoring station (right).

monitoring stations are exchanged on a daily basis during an emergency and at a lower frequency during routine. To make the network suitable for operation during an emergency, redundancy is built-in: all data are collected and available at JRC Ispra, Italy, the German Federal Office for Radiation Protection (BfS) in Freiburg, Germany, and the DG ENER in Luxembourg. This large scale data harmonisation and exchange system for radioactivity measurements is unique in the world.

While the participation to EURDEP by ECURIE Member States is now officially fulfilling their obligation to send off-site environmental monitoring data as specified under the art. $4 \mathrm{~b}$ of Council Decision 87/600 Euratom [1], the participation of non-ECURIE countries is based on a Memorandum of Understanding and on a gentlemen's agreement that data-transmissions will continue during an emergency and with a higher time-resolution.

The Fukushima accident, being very far from the European area, showed once more the vital importance of measurements from HVASs. The maximum air concentration values over Europe were between a factor 1000 to 10000 lower than during the Chernobyl accident and did not result in a detectable increase of the gamma-dose rate values. Therefore the EC asked the EURDEP data-providers to increase the amount of air-concentration data (i.e. measurements from HVASs) during the first weeks of the accident when releases of radionuclides to the atmosphere occurred in Japan.

During March and April 2011 air concentration data were received in the EURDEP data-format from some 12 countries. Another 17 countries made air concentration data available in other formats that had to be converted (some manually) before upload in the EURDEP system. This labour-intensive activity resulted in a unique repository containing most of the air-concentration measurements done in Europe during the first two months of the Fukushima accident. Based on this experience the EC will further motivate data-providers to provide continuously natural and artificial radionuclide airconcentrations, both during routine and emergency. The EC also intends to draft recommendations for further harmonizing air-concentration measurements in Europe.

Future work will focus on extending the number of participating countries, transmitting more on-site meteorological data, expanding to the whole spectrum of sample types foreseen by the Euratom Treaty, refining measurements by applying filters for various natural background components, and, last but not least, globalising the system in collaboration with the IAEA.

\section{ENSEMBLE}

Long-range transport and dispersion models are operationally used in various European countries. Clearly, the various approaches to the simulation of atmospheric dispersion and deposition do not always lead to an unanimous consensus on the forecast: this is directly connected to the differences in the 

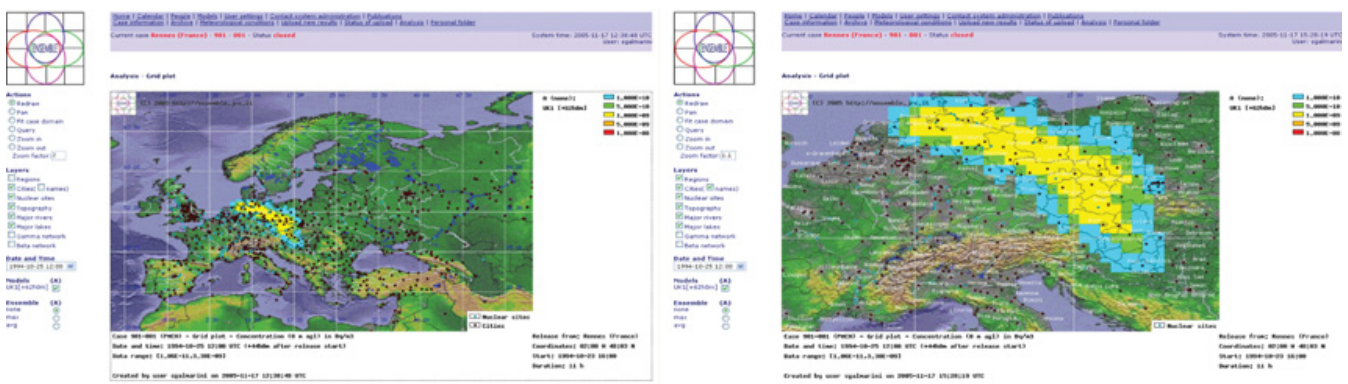

Figure 3. GIS interface (left) and zoom-in capability (right) of the ENSEMBLE system [5].

weather forecast (necessary to predict the dispersion) produced by different weather services and the different approaches to atmospheric dispersion modelling.

Therefore ENSEMBLE has been created with the primary aim of harmonizing the information coming from the various countries and to work out a reconciled and comprehensive European ensemble dispersion forecast. The concept of multi-model ensemble dispersion forecast [2] consists of the qualitative and quantitative analysis of the results produced by various modelling tools on the same case, to produce a composite picture that includes all contributions from the various simulations. This analysis has the advantage of taking into account all possible forecasted scenarios and to determine the level of consensus among the various modelling tools. Within ENSEMBLE the differences in atmospheric dispersion prediction become an asset as they are used to determine the forecast reliability.

More in detail, Ensemble is a web-based system [3] located on a server at JRC Ispra that can collect in real-time the results of 24 atmospheric dispersion models. The model predictions are produced operationally by meteorological offices and radiation protection institutes in Europe, USA and Canada. Model results, produced according to a specific format, can be displayed in real time as single model or combined output by means of ad-hoc statistical treatments in groups (ensembles) for the simultaneous analysis of several forecasts [4]. The modelling groups participating to ENSEMBLE can access the system remotely through a simple web-browser, and each remote user can derive in real time an independent analysis of the information uploaded. Through the ENSEMBLE web site the various model results can be consulted graphically and analysed quantitatively according to ad-hoc statistical procedures, with the aim of quantifying the forecast diversities and prediction reliability. Since the various dispersion forecast are produced according to a pre-defined format, all users have therefore the possibility to access all the information produced by other groups and to compare interactively their model results with those produced in other countries. At the same time since the system is web-based, other users can access the information produced on the dispersion forecast. ENSEMBLE allows each modelling group to access the results of the other 23 models; the decision maker can base countermeasures on a large set of model results rather than only those produced at national level.

The coupling of ENSEMBLE with the EURDEP database represents the most relevant of the latest improvements of the ENSEMBLE system [5]. In case of a nuclear accident or any other event that would imply the dispersion of radioactive contaminants, every ENSEMBLE user will have the possibility to consult the EURDEP database and to display any measurement collected in real-time by the European network and use it for evaluating a model or an ensemble of models. Since all the models produce concentration predictions the comparison can only be qualitative but yet very revealing. What makes the monitoring data most relevant is the availability and accessibility of a large number of model results. As anticipated earlier, EURDEP foresees in the future an increase in the number of concentration measurements performed throughout the European domain. When more of these monitoring data will be available, ENSEMBLE will also become a valuable tool for the application of data assimilation techniques within atmospheric dispersion models. 
This coupling of ENSEMBLE with EURDEP was the starting point for a more radical restructuring of the system. All the new facilities were added to facilitate the consultation of both model results and data values and the access to a much wider set of information. The main new features added to the system are:

- modified graphical display of model results and monitoring data by means of a GIS interface (Figure 3),

- adaptability of space domain in terms of dislocation and resolution,

- possibility for each modelling group to submit dispersion predictions relating to multiple nuclides.

\section{CONCLUSIONS}

During the early phase of a large-scale accident with release of radioactivity to the atmosphere, it is essential to have threatened countries notified as soon as possible in the most efficient way: information needs to be transmitted with fast and secure systems, in a harmonized way and languageindependent. ECURIE is the official EC early notification system that alerts the 27 EU Member States, Switzerland, Croatia and the Republic of Macedonia. ECURIE is also designed to provide continuous, detailed information during such an accident, for example planned countermeasures and public information releases. In 2012 the ECURIE software will migrate from dedicated CoDecS stations to the WebECURIE web-site. Further developments will involve the improvement of the harmonisation with the IAEA notification system.

To transmit and share environmental monitoring data in real-time, the EURDEP system was designed to facilitate the transmission of large datasets, thereby taking away the burden from national crisis centres to manually report and transmit such data under ECURIE. Noteworthy is the fact that the collected data can be analysed and accessed by means of a simple GIS interface that is available via the EURDEP web site. Currently EURDEP is limited to the European area, but from 2012 onwards the system will be globalized in collaboration with the IAEA.

The ENSEMBLE module intercompares contamination predictions by assessing and mapping the results of major worldwide dispersion models. The coupling of ENSEMBLE with the EURDEP database represents the most important of the latest improvements of the ENSEMBLE system.

The three systems are currently bridged only loosely, as this strategy facilitates the development and testing of the concepts and devices; however in future, they should evolve into a more integrated system. This would allow decision makers to receive, during the early phase of an emergency response, critical information regarding the current status and expected evolution of the accident, the radiological situation as observed by the monitoring networks and the atmospheric dispersion forecasts in a more manageable and coherent way.

\section{Acknowledgments}

The authors are grateful to all direct and indirect contributors and national data providers to the above-mentioned European networks, for without their help, this work could not have been possible. The authors also wish to thank their colleague Tore Tollefsen whose meticulous review of the manuscript substantially improved its readability.

\section{References}

[1] Council Decision 87/600 of 14 December 1987 on Community arrangements for the early exchange of information in the event of a radiological emergency (OJ L371 of 30.12.1987).

[2] Galmarini, S., Bianconi, R. Bellasio, R. and Graziani, G., J Environmental Radioactivity 57 (2001) 203-219.

[3] Bianconi R., Galmarini S. and Bellasio R., J Environmental Modelling and Software 19 (2004) 401-411. 
[4] Galmarini S., Bianconi R. Klug W., Mikkelsen T., Addis R., Andronopoulos S., Astrup P., Baklanov A., Bartniki J., Bartzis J.C., Bellasio R., Bompay F., Buckley R., Bouzom M., Champion H., D’Amours R., Davakis E., Eleveld H., Geertsema G. T., Glaab H., Kollax M., Ilvonen M., Manning A., Pechinger U., Persson C., Polreich E., Potemski S., Prodanova M., Saltbones J., Slaper H., Sofiev M.A., Syrakov D., Sørensen J.H., Van der Auwera L., Valkama I. and Zelazny R., Atmospheric Environment 38 (2004) 4607-4617.

[5] Galmarini S., Bianconi R., de Vries G. and Bellasio R., J. Environmental Radioactivity 99 (2008) 1233-1241. 